IN THE NEWS

Time drags when you're

having none

A recent study in

Psychopharmacology

Bulletin shows that

nicotine withdrawal

impairs time perception.

Laura Cousino Klein and

colleagues from Penn

State University suggest

that the tendency for

smokers to overestimate

the duration of short

intervals after just 24 hours

without a cigarette

contributes to the anxiety

and concentration

difficulties that beset those

who try to kick the habit

In a small experiment

with 44 subjects, Klein's

team had smokers and

non-smokers assess the

duration of a 45-second

interval. Prior to nicotine

withdrawal, the smokers' responses did not differ significantly from those of the non-smokers - both groups made similarly accurate estimations, with an error range of about 5 seconds. However, after foregoing tobacco for

24 hours, smokers over-

estimated the amount of

time that had elapsed, by

$50 \%$ on average. "We had

some people [who]

thought it was three

minutes", Klein told The

Associated Press.

To smokers who have

tried to quit, these

findings come as no

surprise. A student at

Penn State in the throes of

withdrawal lamented that

"When I'm sitting, when

l'm bored ... one minute

passes and it seems like

five" (Post-Gazette, USA,

14 May 2003). But

encouragement to tough

it out came from a spokes-

woman for the UK charity

Action for Smoking and

Health who pointed out

that "These sort of feelings

are over very quickly.

Nicotine leaves the blood

within $\mathbf{4 8}$ hours so it should

get much easier after two

days" (BBC News, UK,

10 May 2003). Smokers be

warned - according to the

new study, those two days

will feel more like three.

Suzanne Farley
SYNAPTIC PHYSIOLOGY

\section{Kiss-me-quick synapses}

Wouldn't it be easier to extract the secrets of the synapse if we could see what is going on there? For example, if we could just watch a synaptic vesicle fusing with the membrane and then being recycled, we could finally answer some of the questions about how the recycling is accomplished. Two papers in Nature use fluorescent markers to achieve just that, and reach complementary conclusions about vesicle recycling at small synapses.

Our understanding of synaptic exocytosis - the process by which individual vesicles fuse with the cell membrane and release their loads of neurotransmitter into the synaptic cleft - has come on in leaps and bounds over recent years. But what happens next is more of a mystery. It is generally thought that most vesicles collapse into the presynaptic membrane before their components are retrieved, but another school of thought suggests that some vesicles do not collapse; instead, they fuse transiently with the membrane and open a fusion pore, before closing the pore and being recycled as a unit. This mechanism, which is called 'kiss-and-run' exocytosis, receives strong support from the two new papers.

In the first study, Gandhi and Stevens infected hippocampal neurons with a virus carrying the gene for synaptophluorin - a pH-sensitive marker that fluoresces when the inside of a vesicle is exposed to the external medium. When a cultured neuron is stimulated, the fluorescence rises as vesicles fuse with the membrane and then falls back as they are endocytosed. By studying the time course of these events, the authors were able to show that vesicles undergo three distinct types of exocytosis. In the first, which Gandhi and Stevens specifically call kiss-andrun, vesicles are recycled quickly (in less than a second) and the fusion pore that opens is selective - for example, the buffer Tris can enter the vesicles but another buffer, HEPES, cannot.

The second type of fusion was slower, with vesicles being recycled after 8-21 seconds, and is termed 'compensatory' endocytosis. In the third, 'stranded' vesicles seem to remain fused with the membrane for more than 45 seconds, although it seems that their endocytosis can be triggered by subsequent action potentials.

Aravanis et al. used a different technique, in which vesicle membranes are labelled with a fluorescent dye (FM1-43) that is released when exocytosis is stimulated in dye-free medium. The drop in fluorescence can be seen when exocytosis is triggered by stimulation of cultured neurons. Most vesicles lost only part of their fluorescence when they fused with the membrane, supporting the idea that they released the dye through kiss-and-run exocytosis rather than by complete collapse into the cell membrane. And, as pointed out by Rizzoli and Betz in an accompanying News and Views article, the time-course of dye release indicates that

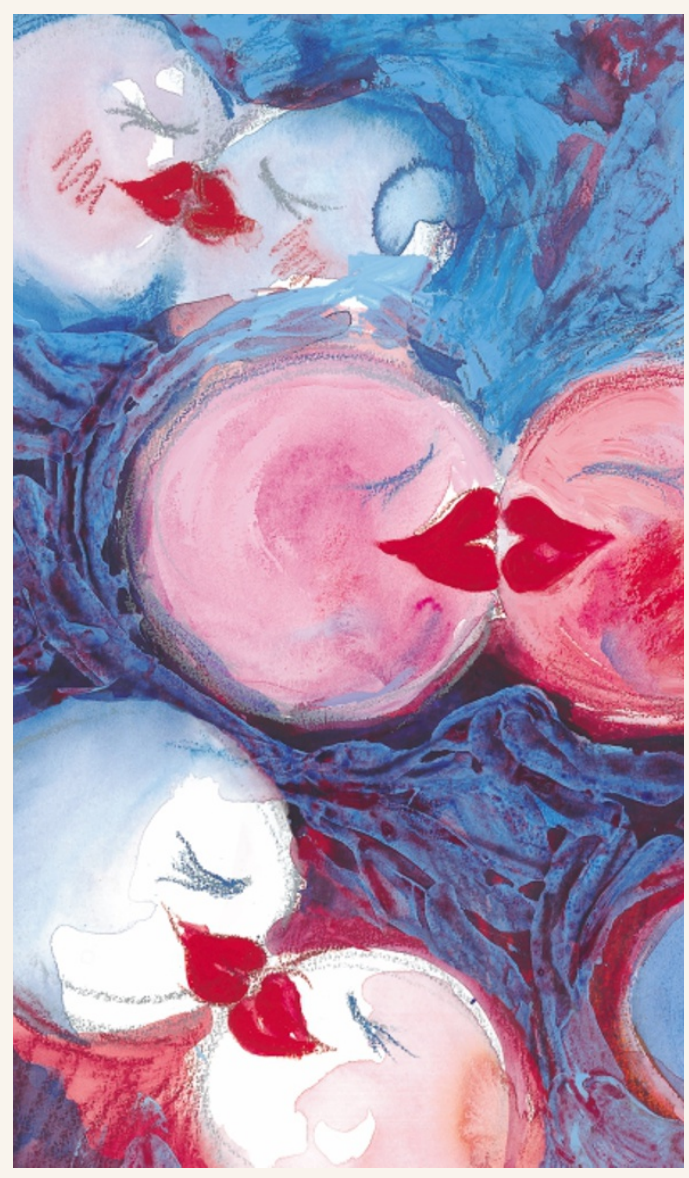

these fusion events correspond to the 'compensatory' endocytosis described by Gandhi and Stevens, rather than to their very fast kiss-and-run events.

So these two studies provide compelling evidence that most vesicles in small hippocampal synapses fuse by a kiss-and-run type of exocytosis, rather than by collapsing into the presynaptic membrane. This finding contrasts with previous studies showing that vesicles collapse before being recycled slowly; but many of the earlier studies used different types of synapses with large numbers of vesicles. The techniques used in these two papers should allow future work to resolve such discrepancies, and to look deeper into the mechanisms and control of synaptic recycling.

Rachel Jones

(0)) References and links

ORIGINAL RESEARCH PAPERS Gandhi, S. P. \& Stevens, C. F. Three modes of synaptic vesicular recycling revealed by single-vesicle imaging Nature 423, 607-613 (2003) | Aravanis, A. M. et al. Single synaptic vesicles fusing transiently and successively without loss of identity. Nature 423, 643-647 (2003)

FURTHER READING Rizzoli, S. O. \& Betz, W. J. All change at the synapse. Nature 423, 591-592 (2003) |Rizo, J. \& Südhof T. C. SNARES and Munc18 in synaptic vesicle fusion. Nature Rev Neurosci. 3, 641-653 (2002) 